\title{
Normas de publicação
}

\section{Normas RFO}

A RFO UPF é uma publicação quadrimestral dirigida à classe odontológica que tem por objetivo disseminar e promover o intercâmbio de informações científicas, indexada nas bases de dados da BBO (Bibliografia Brasileira de Odontologia), Lilacs (Literatura Latino-Americana e do Caribe em Ciências da Saúde), Latindex (Sistema Regional de Información en Línea para Revistas Científicas de América Latina, el Caribe, España y Portugal) e Rev@odonto.

A RFO UPF divulga artigos inéditos de investigação científica; resumos de teses, dissertações e monografias; relatos de casos clínicos e artigos de revisão sistemática que representam contribuição efetiva para a área do conhecimento odontológico.

Os manuscritos deverão ser encaminhados somente via submissão on-line, utilizando o website http://www.upf.br/seer/index.php/rfo.

\section{Normas gerais}

a) Os conceitos e informações emitidos no texto são de inteira responsabilidade do(s) autor(es), não refletindo, necessariamente, a opinião do Conselho Editorial e Científico da revista.

b) Todos os manuscritos serão submetidos, inicialmente, à apreciação dos editores de área, e, se adequados à revista, serão submetidos a um Conselho Científico; posteriormente os autores serão notificados pelo editor, tanto no caso de aceitação do artigo como da necessidade de alterações e revisões ou rejeição do trabalho. Eventuais modificações na forma, estilo ou interpretação dos artigos só ocorrerão após prévia consulta e aprovação por parte do(s) autor(es).

c) A correção das provas tipográficas estará a cargo dos autores.

d) Cada trabalho publicado dará direito a um exemplar impresso da revista. Por solicitação do(s) autor(es) poderão ser fornecidos exemplares adicionais, sendo-lhes levado a débito o respectivo acréscimo.

e) Serão aceitos para revisão manuscritos com, no máximo, seis autores.

\section{Apresentação dos originais}

Os artigos destinados à RFO UPF deverão ser redigidos em português ou em inglês, de acordo com o estilo dos Requisitos Uniformes para Originais submetidos a Revistas Biomédicas, conhecido como Estilo de Vancouver, versão publicada em outubro de 2005, elaborada pelo Comitê Internacional de Editores de Revistas Médicas (ICMJE) e baseado no padrão Ansi, adaptado pela U.S. National Library of Medicine.

O texto deverá ser digitado em fonte Times New Roman tamanho 12, papel tamanho A4, com espaço duplo e margens de $3 \mathrm{~cm}$ de cada lado, perfazendo um total de, no máximo, vinte páginas, incluindo tabelas, quadros, esquemas, ilustrações e respectivas legendas. As páginas deverão ser numeradas com algarismos arábicos no ângulo superior direito da folha. O título do artigo (em português e em inglês), assim como os subtítulos que o compõem deverão ser impressos em negrito. Deverão ser grafadas em itálico palavras e abreviaturas escritas em outra língua que não a portuguesa, como o latim (ex: in vitro) e o inglês (ex: single bond). As grandezas, unidades, símbolos e abreviaturas devem obedecer às normas internacionais ou, na ausência dessas, às normas nacionais correspondentes.

Qualquer trabalho que envolva estudo com seres humanos, incluindo-se órgãos e/ou tecidos separadamente, bem como prontuários clínicos ou resultados de exames clínicos, deverá estar de acordo com a resolução 196/96 do Conselho Nacional de Saúde e seus complementos, e ser acompanhado da aprovação de uma Comissão de Ética em Pesquisa. Não devem ser utilizados no material ilustrativo nomes ou iniciais dos pacientes, tampouco registros hospitalares. Nos experimentos com animais, devem ser seguidos os guias da Instituição dos
Conselhos Nacionais de Pesquisa sobre o uso e cuidados dos animais de laboratório, e o estudo deve ser acompanhado da aprovação da Comissão de Ética no Uso de Animais (Ceua).

No caso de trabalhos aceitos para publicação totalmente em inglês, correrá por conta dos autores o custo de revisão gramatical, com tradutor indicado pela Coordenação de Editoração do periódico. O custo da revisão gramatical da língua inglesa será repassado aos autores. A submissão de um manuscrito em língua inglesa à RFO-UPF implica a aceitação prévia dessa condição. O mesmo é válido para a revisão gramatical dos abstracts.

\subsection{Composição dos manuscritos}

Na elaboração dos manuscritos deverá ser obedecida a seguinte estrutura:

\section{a) página de rosto}

- título do manuscrito no primeiro idioma (que deve ser conciso, mas informativo);

- título do manuscrito no segundo idioma (idem ao anterior);

- nome(s) do(s) autor(es) por extenso, com seu grau acadêmico mais alto e sua filiação institucional (se houver), departamento, cidade, estado e país;

- nome do(s) departamento(s) ou instituição(ões) aos quais o trabaIho deve ser atribuído;

- nome, endereço e e-mail do autor responsável pela correspondência sobre o original.

\section{b) resumo e palavras-chave}

O resumo deve ser estruturado e apresentar concisamente, em um único parágrafo, os objetivos do estudo ou investigação, procedimentos básicos (seleção da amostra, métodos analíticos), principais achados (dados específicos e sua significância estatística, se possível) e as principais conclusões, enfatizando aspectos novos e importantes do estudo ou das observações. Não deve conter menos de 150 e mais de 250 palavras. Deve apresentar as seguintes subdivisões: objetivo, métodos, resultados e conclusão (para investigações científicas); objetivo, relato de caso e considerações finais (para relatos de caso); e objetivos, revisão de literatura e considerações finais (para revisão de literatura). Abaixo do resumo, fornecer, identificando como tal, 3 a 5 palavras-chave ou expressões que identifiquem o conteúdo do trabaIho. Para a determinação dessas palavras-chave, deve-se consultar a lista de "Descritores em Ciências da Saúde - DeCS", elaborada pela Bireme, e a de "Descritores em Odontologia - DeOdonto", elaborada pelo SDO/FOUSP.

\section{c) abstract e keywords}

Idem ao item anterior. Sua redação deve ser paralela à do resumo. d) texto

No caso de investigações científicas, o texto propriamente dito deverá conter os seguintes capítulos: introdução, materiais e método, resultados, discussão, conclusão e agradecimentos (quando houver).

No caso de artigos de revisão sistemática e relatos de casos clínicos, pode haver flexibilidade na denominação desses capítulos.

- Introdução: estabelecer o objetivo do artigo e apresentar as razões para a realização do estudo. Citar somente as referências estritamente pertinentes e não incluir dados ou conclusões do trabalho que está sendo relatado. A hipótese ou objetivo deve ser concisamente apresentada no final dessa seção. Extensas revisões de literatura devem ser evitadas e substituídas por referências aos trabalhos bibliográficos mais recentes, nos quais certos aspectos e revisões já tenham sido apresentados.

- Materiais e método: identificar os materiais, equipamentos (entre parênteses dar o nome do fabricante, cidade, estado e país de fabricação) e procedimentos em detalhes suficientes para permitir 
que outros pesquisadores reproduzam os resultados. Dar referências de métodos estabelecidos, incluindo métodos estatísticos; descrever métodos novos ou substancialmente modificados, dar as razões para usá-los e avaliar as suas limitações. Identificar com precisão todas as drogas e substâncias químicas utilizadas, incluindo nome(s) genérico(s), dose(s) e via(s) de administração.

- Resultados: devem ser apresentados em sequência lógica no texto, nas tabelas e nas ilustrações com o mínimo possível de discussão ou interpretação pessoal. Não duplicar dados em gráficos e tabelas. Não repetir no texto todas as informações das tabelas e ilustrações (enfatizar ou resumir informações importantes).

- Discussão: deve restringir-se ao significado dos dados obtidos, evitando-se hipóteses não fundamentadas nos resultados, e relacioná-los ao conhecimento já existente e aos obtidos em outros estudos relevantes. Enfatizar os aspectos novos e importantes do estudo. Não repetir em detalhes dados já citados nas seções de introdução ou resultados. Incluir implicações para pesquisas futuras.

- Conclusão: deve ser associada aos objetivos propostos e justificada nos dados obtidos. A hipótese do trabalho deve ser respondida.

- Agradecimentos: citar auxílio técnico, financeiro e intelectual que porventura possam ter contribuído para a execução do estudo.

- Formas de citação no texto: no texto, utilizar o sistema numérico de citação, no qual somente os números-índices das referências, na forma sobrescrita, são indicados. Números sequenciais devem ser separados por hífen; números aleatórios devem ser separados por vírgula. Evitar citar os nomes dos autores e o ano de publicação. Somente é permitida a citação de nomes de autores (seguidos de número-índice e ano de publicação do trabalho) quando estritamente necessário, por motivos de ênfase.

Exemplos de citação de referências bibliográficas no texto: variável ${ }^{3}$

- "...manifesta-se como uma dor constante, embora de intensidade

- "Entre as possíveis causas da condição estão citados fatores psicogênicos, hormonais, irritantes locais, deficiência vitamínica, fármacos e xerostomia ${ }^{1-4,6,9,15}$.

- Um autor: Field ${ }^{4}$ (1995)...;

- Dois autores: Feinmann e Peatfield ${ }^{5}$ (1995)...

- Mais de dois autores: Sonis et al. ${ }^{8}(1995) \ldots ;$

e) referências

As referências devem ser ordenadas no texto consecutivamente na ordem em que foram mencionadas, numeradas e normatizadas de acordo com o Estilo Vancouver, conforme orientações fornecidas pelo International Committee of Medical Journal Editors no "Uniform Requirements for Manuscripts Submitted to Biomedical Journals" (http:// www. icmje.org). Os títulos de periódicos devem ser abreviados de acordo com o "List of Journals Indexed in Index Medicus" (http://www. $\mathrm{nlm.nih.gov/tsd/serials/lji.html)} \mathrm{e} \mathrm{impressos} \mathrm{sem} \mathrm{negrito,} \mathrm{itálico} \mathrm{ou} \mathrm{gri-}$ fo, devendo-se usar a mesma apresentação em todas as referências. Os sobrenomes dos autores devem ser seguidos pelos seus prenomes abreviados sem ponto ou vírgula. Usar a vírgula somente entre os nomes dos diferentes autores.

Nas publicações com até seis autores, citam-se todos; nas publicações com sete ou mais autores, citam-se os seis primeiros e, em seguida, a expressão latina "et al.". Incluir ano, volume, número (fascículo) e páginas do artigo logo após o título do periódico. Deve-se evitar a citação de comunicações pessoais, trabalhos em andamento e os não publicados; caso seja estritamente necessária sua citação, não devem ser incluídos na lista de referências, mas citados em notas de rodapé. A exatidão das referências bibliográficas é de responsabilidade dos autores.

\section{Exemplos de referências}

Livro:

Netter FH. Atlas de anatomia humana. 2. ed. Porto Alegre:Artes Médicas Sul; 2000.

Livro em suporte eletrônico:

Wothersponn AC, Falzon MR, Isaacson PG. Fractures: adults and old people [monograph on CD-ROM]. 4. ed. New York: Lippincott-Raven; 1998.

Ueki N, Higashino K, Ortiz-Hidalgo CM. Histopathology [monograph online].

Houston: Addison Books; 1998. [cited Jan 27]. Available from: URL: http://www.hist.com/dentistry.
Capítulo de livro:

Estrela C, Bammann LL. Medicação intracanal. In: Estrela C, Figueiredo JAP. Endodontia. Princípios biológicos e mecânicos. 2. ed. São Paulo: Artes Médicas; 1999. p. 571-653.

Capítulo de livro em suporte eletrônico:

Chandler RW. Principles of internal fixation. In: Wong DS, Fuller LM. Prosthesis [monograph on CD-ROM]. 5. ed. Philadelphia: Saunders; 1999.

Tichemor WS. Persistent sinusitis after surgery. In: Tichenor WS. Sinusitis: treatment plan that works for asthma and allergies too [monograph online]. New York: Health On the Net Foundation; 1996. [cited 1999 May 27]. Available from: URL: http://www.sinuses.com/postsurg. htm.

Editor(es) ou compilador(es) como autor(es) de livros:

Avery JK, editor. Oral development and histology. 2. ed. New York: Thieme Medical Publishers; 1994.

Organização ou sociedade como autor de livros:

American Dental Association and American Academy of Periodontology. Introduce dentist to new time saving periodontal evaluation system. Washington: The Institute; 1992.

Artigo de periódico:

Barroso LS, Habitante SM, Silva FSP. Estudo comparativo do aumento da permeabilidade dentinária radicular quando da utilização do hipoclorito de sódio. J Bras Endod 2002; 11(3):324-30.

McWhinney S, Brown ER, Malcolm J, VillaNueva C, Groves $B M$, Quaife RA, et al. Identification of risk factors for increased cost, charges, and length of stay for cardiac patients. Ann Thorac Surg 2000;70(3):702-10.

Artigo de periódico em suporte eletrônico:

Nerallah LJ. Correção de fístulas pela técnica de bipartição vesical. Urologia On line [periódico online] 1998 [citado 1998 Dez 8]; 5(4):[telas]. Disponível em URL: http://www.epm.br/cirurgia/uronline/ ed0798/fistulas.htm.

Chagas JCM, Szejnfeld VL, Jorgetti V, Carvalho AB, Puerta EB. A densitometria e a biópsia óssea em pacientes adolescentes. Rev Bras Ortop [periódico em CD-ROM] 1998; 33(2).

Artigo sem indicação de autor:

Ethics of life and death. World Med J 2000; 46:65-74.

Organização ou sociedade como autor de artigo:

World Medical Association Declaration of Helsinki. Ethical principles for medical research involving human subjects. Bull World Health Organ 2001; 79:373-4.

Volume com suplemento:

Shen HM, Zhang QF. Risk assessment of nickel carcinogenicity and occupational lung cancer. Environ Health Perspect 1994;102 Suppl 1:275-82.

Fascículo sem indicação de volume:

Graf R. Hip sonography: how reliable? Dynamic versus static examination. Clin Orthop 1992; (218):18-21.

Sem volume ou fascículo:

Brown WV. The benefit of aggressive lipid lowering. J Clin Practice 2000:344-57.

Resumo:

Clement J, de Bock R. Hematological complications [abstract]. Quintessence Int 1999; 46:1277.

Errata:

White P. Doctors and nurses. Let's celebrate the difference between doctors and nurses. [published erratum in $\mathrm{Br}$ Med ] 2000;321(7264):835]. Br Med J 2000; 321(7262):698.

Artigo citado por outros autores - apud:

O'Reilly M, Yanniello GJ. Mandibular growth changes and maturation of cervical vertebrae. A longitudinal cephalometric study (1988) apud Mito T, Sato K, Mitani H. Predicting mandibular growth potential with cervical vertebral bone age. Am J Orthod Dentofacial Orthop 2003; 124(2):173-7. 


\section{Dissertações e teses:}

Araújo TSS. Estudo comparativo entre dois métodos de estimativa da maturação óssea [Dissertação de Mestrado]. Piracicaba: Faculdade de Odontologia de Piracicaba da Unicamp; 2001.

Dissertações e teses em suporte eletrônico:

Ballester RY. Efeito de tratamentos térmicos sobre a morfologia das partículas de pó e curvas de resistência ao CREEP em função do conteúdo de mercúrio, em quatro ligas comerciais para amálgama [Tese em CD-ROM]. São Paulo: Faculdade de Odontologia da USP; 1993.

Trabalho apresentado em evento:

Cericato GO, Cechinato F, Moro G, Woitchunas FE, Cechetti D, Damian MF. Validade do método das vértebras cervicais para a determinação do surto de Crescimento Puberal. In: 22a Reunião Anual da Sociedade Brasileira de Pesquisa Odontológica: 2005; Águas de Lindóia. Anais. Brazilian Oral Research; 2005. p. 63

Trabalho de evento em suporte eletrônico:

Gomes SLR. Novos modos de conhecer: os recursos da Internet para uso das Bibliotecas Universitárias [CD-ROM]. In: 10º Seminário Nacional de Bibliotecas Universitárias; 1998 Out 25-30; Fortaleza. Anais. Fortaleza: Tec Treina; 1998.

Barata RB. Epidemiologia no século XXI: perspectivas para o Brasil. In: 4º Congresso Brasileiro de Epidemiologia [online]; 1998 Ago 1-5; Rio de Janeiro. Anais eletrônicos. Rio de Janeiro: ABRASCO;1998 [citado 1999 Jan 17]. Disponível em URL: http://www.abrasco.com.br/ epirio98/.

Documentos legais:

Brasil. Portaria n. 110, de 10 de março de 1997. Diário Oficial da República Federativa do Brasil, Brasília, 18 mar 1997, seção 1, p. 5332.

f) tabelas, quadros, esquemas e gráficos

Devem ser inseridos ao longo do texto, logo após sua citação no mesmo. Devem ser numerados consecutivamente em algarismos arábicos. As legendas das tabelas e dos quadros devem ser colocadas na parte superior dos mesmos e, quando for necessário, incluir logo abaixo desses uma listagem dos símbolos, abreviaturas e outras informações que facilitem sua interpretação. As legendas de esquemas e de gráficos devem ser colocadas na parte inferior dos mesmos. Todas as tabelas e todos os quadros, esquemas e gráficos, sem exceção, devem ser citados no corpo do texto.

Obs.: Os gráficos deverão ser considerados como "figuras" e constar da sequência numérica juntamente com as imagens.

g) imagens (fotografias, radiografias e microfotografias)

Imagens digitais deverão ser submetidas em tamanho e resolução adequados (300 dpi). Não serão aceitas imagens digitais artificialmente "aumentadas" em programas computacionais de edição de imagens. A publicação de imagens coloridas é de opção dos autores que devem manifestar seu interesse caso o manuscrito seja aceito para publicação. O custo adicional da publicação das imagens coloridas é de responsabilidade do(s) autor(es).

Todas as imagens, sem exceção, devem ser citadas no texto. As microfotografias deverão apresentar escala apropriada.

Poderão ser submetidas um máximo de oito imagens, desde que sejam necessárias para a compreensão do assunto. 


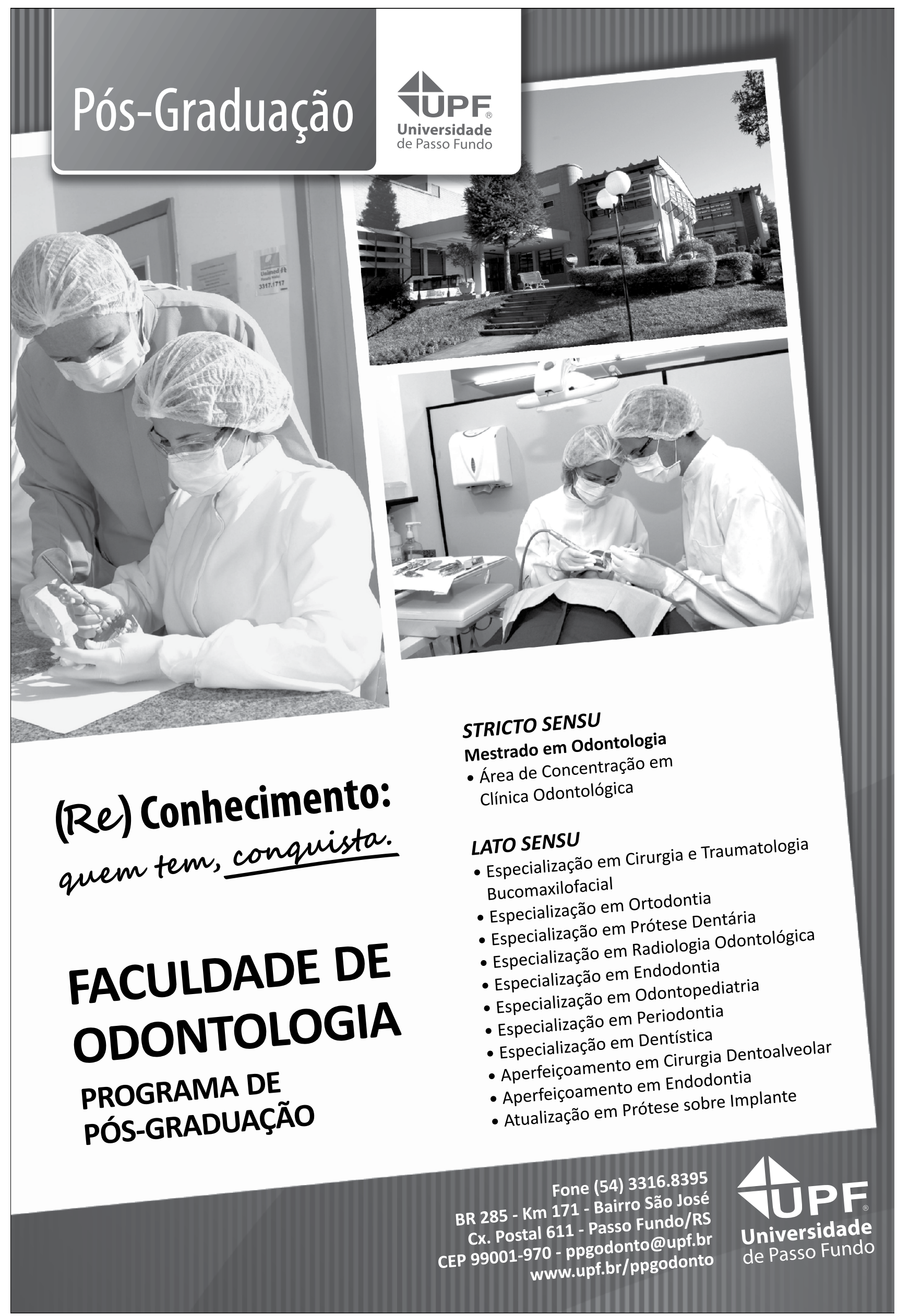

